\title{
ABSORCIÓN POR OZONO Y DISPERSIÓN POR AIRE DE LA RADIACIÓN SOLAR ULTRAVIOLETA B
}

\section{OZONE ABSORPTION AND PURE AIR SCATTERING OF SOLAR ULTRAVIOLETA B RADIATION}

\author{
Julio César González Navarrete¹, Julián Salamanca²
}

\begin{abstract}
${ }^{1}$ Magister en Ciencias-Física. Dirección de Investigación, Fundación Universitaria Los Libertadores. Correo Electrónico: jucegonza3@ hotmail.com. 2 Ph.D. en Física Aplicada. Dirección de Investigación, Fundación Universitaria Los Libertadores. Correo Electrónico: jusalama@gmail.com.
\end{abstract}

Rev. U.D.C.A Act. E Div. Cient. 14(2): 151 - 159, 2011

\section{RESUMEN}

Para los casos eventuales de falta de información experimental, se presenta una alternativa para poder disponer de datos confiables y validables de los coeficientes de absorción por ozono y de dispersión por aire puro de la radiación solar ultravioleta B (radiación UV-B); dichos coeficientes son requeridos para la estimación de la columna total de ozono al aplicar la espectrofotometría Dobson. La alternativa propuesta tiene que ver con el uso cuidadoso de interpolación, aplicada a series de datos, debidamente validados. Se plantea un ensayo de validación indirecta aplicando la metodología Dobson a la información obtenida de esta manera y así determinar el ozono total.

Palabras clave: Radiómetros, ozono, radiación solar, radiación ultravioleta, absorción, dispersión.

\section{SUMMARY}

For eventual cases of lack of experimental data, an alternative in order to have reliable and verifiable results for both ozone absorption and pure air dispersion coefficients of solar ultraviolet $B$ radiation (UV-B) is shown; these coefficients are required to estimate ozone total column in Dobson spectrophotometry. The proposed alternative has to do with the careful use of interpolation applied to sets of properly validated data. We present an indirect validation test using Dobson methodology to the information obtained in this manner and thus determine the total ozone.

Key words: Radiometer, ozone, solar radiation, ultraviolet radiation, absorption, dispersion.

\section{INTRODUCCIÓN}

La radiación solar, en su paso por la atmósfera, es sometida a varios fenómenos que atenúan su intensidad de manera espectralmente selectiva. Estos procesos son esencialmente:

- La absorción selectiva, ocasionada por diferentes gases y por el vapor de agua.

- La dispersión molecular o de Rayleigh, también generada por los gases y por el vapor de agua.

- La dispersión y la absorción formada por los aerosoles, denominada turbidez o turbiedad (Iqbal, 1983).

Para el tratamiento matemático simultáneo de estos fenómenos, se asume que se dan las condiciones para aplicar la ley de Beer-Bouguer-Lambert en su forma espectral y para diferentes alturas del Sol, en la forma,

$$
\mathrm{I}_{\mathrm{n} \lambda}=\mathrm{I}_{\mathrm{on} \lambda} \mathrm{e}^{-\mathrm{k} \lambda \mathrm{m}}
$$

con:

$I_{n}$, la irradiancia solar directa de longitud de onda; $\lambda$, que incide en la superficie terrestre después de atravesar la atmósfera; $\mathrm{I}_{\mathrm{on} \wedge}$, la irradiancia solar extraterrestre con longitud de onda $\lambda ; k_{\lambda}$, el coeficiente de atenuación o extinción de la radiación a la misma longitud de onda y $\mathrm{m}$, el camino óptico o masa óptica atravesada por la radiación en su camino por la atmósfera.

Cada especie química que se halla en la atmósfera contribuye, de forma característica, en el incremento de la atenuación de la radiación solar. Con el fin de estimar la radiación solar que llega a la superficie, se han desarrollado modelos semiempíricos, que involucran los fenómenos, en los que dichas especies intervienen (Boscá, 1995); en varios 
de tales modelos, el exponente de la Ecuación 1, se expresa matemáticamente como:

$\mathrm{k}_{\lambda} \mathrm{m}=\mathrm{k}_{\mathrm{r} \lambda} \mathrm{m}_{\mathrm{r}}+\mathrm{k}_{\mathrm{o} \lambda} \mathrm{m}_{\mathrm{o}}+\mathrm{k}_{\mathrm{w} \lambda} \mathrm{m}_{\mathrm{w}}+\mathrm{k}_{\mathrm{g} \lambda} \mathrm{m}_{\mathrm{g}}+\mathrm{k}_{\mathrm{a} \lambda} \mathrm{m}_{\mathrm{a}}$

En general, se asume que todos los procesos de atenuación de la radiación solar son linealmente independientes para cada longitud de onda (Liou, 2002). Los principales procesos de atenuación están representados por: coeficiente de dispersión de Rayleigh $\mathrm{k}_{\mathrm{r} \lambda}$, que explica la atenuación de radiación, debida a dispersión por partículas de tamaño menor que la longitud de onda de la radiación incidente; coeficiente de absorción de la radiación, debida al ozono $\mathrm{k}_{\mathrm{o}}$, Ocasionada por la presencia de este compuesto en un estrato de la atmósfera; coeficiente de absorción, debida al vapor de agua $\mathrm{k}_{\mathrm{w} \lambda}$, relacionada con la humedad relativa y la temperatura de la superficie; coeficiente de absorción, debida a los gases uniformemente mezclados $\mathrm{k}_{\mathrm{g}}$, por ejemplo, $\mathrm{CO}_{2}$ y $\mathrm{O}_{2}$, cuya razón de mezcla permanece constante hasta, aproximadamente, $90 \mathrm{~km}$ sobre la superficie terrestre y, finalmente, el coeficiente que muestra los efectos de absorción y de dispersión de los aerosoles $\mathrm{k}_{\mathrm{a}}$. Es pertinente anotar que cada coeficiente de atenuación debe involucrar, a su vez, la masa óptica correspondiente $\mathrm{m}$; que estos coeficientes tienen unidades de inverso de longitud y que la Ecuación 2 excluye los efectos de absorción de contaminantes. Si en la Ecuación 1 los valores de la irradiancia extraterrestre y terrestre y los coeficientes de absorción son conocidos, es posible determinar, por ejemplo, la masa óptica del ozono en columna vertical; sin embargo, no siempre se dispone de los valores experimentales específicos de absorción de la radiación por ozono o de dispersión por el aire, lo cual, representa frecuentemente un escollo para la determinación de la columna total del ozono en las metodologías, cuyo fundamento sea la Ecuación 1.

Dentro del conjunto de las interacciones que ocurren entre la radiación solar ultravioleta (R-UV) y el ozono atmosférico, se destacan las que se presentan en el rango espectral de 300 a $340 \mathrm{~nm}$, que forma parte de la banda Huggins (García, 2009); tales interacciones son aprovechadas para determinar la columna total de ozono, entre otras, por las metodologías espectrofotométricas, particularmente, de Dobson y de Brewer (Staehelin et al. 2003), que se fundamentan en la Ecuación 1. En este trabajo, se presentarán y se discutirán algunos criterios relativos al uso de datos obtenidos por interpolación de los coeficientes de absorción espectral de la R-UV, por parte del ozono atmosférico y de la dispersión, por parte de los componentes del aire puro, en el mismo rango espectral de 300 a $340 \mathrm{~nm}$; este rango espectral es justamente el recomendado para ser utilizado en las metodologías mencionadas. Los valores de los coeficientes en cuestión, pilar de este trabajo, fueron obtenidos en laboratorios por los diferentes grupos de investigadores, referenciados a lo largo de este artículo.

La absorción espectral de la radiación, se determina por el coeficiente de absorción que, para el ozono, depende, en gran parte, de la temperatura (Grebenshchikov et al. 2007); tal dependencia, se presenta por que la sección transversal de absorción del ozono, que es directamente proporcional al coeficiente de absorción, muestra también dependencia espectral y térmica (Staehelin et al. 2003; Bernhard et al. 2005b)

La R-UV que no es absorbida por el ozono es susceptible de ser dispersada espectralmente por los componentes atmosféricos y el indicador del nivel de dispersión, se expresa mediante el coeficiente de dispersión (Goody, 1964). Se han reportado mediciones de estos coeficientes en condiciones controladas de laboratorio, utilizando algunos valores espectrales discretos de R-UV, en el rango entre 300 y 340nm (Bernhard et al. 2005b; Murad \& Tabocchini, 1976); sin embargo, no se dispone de mediciones de dichos coeficientes para todas las longitudes de onda en el rango espectral mencionado $y$, con mucha frecuencia, se requiere conocer valores específicos.

Este trabajo expone una opción de utilizar los valores experimentales existentes para estimar, con alto grado de seguridad, valores aún no medidos o no reportados; esta opción ha sido empleada por varios investigadores en otros rangos espectrales (Iqbal, 1983).

El interés de este trabajo radica, esencialmente, en que dentro del rango UV-B la absorción de la radiación por parte del ozono decae, abruptamente, a medida que aumenta la longitud de onda; esta situación sugiere, el empleo de absorción diferencial de la radiación entre pares de longitudes de onda, lo cual, desde los puntos de vista físico y matemático, es aprovechado para estimar la columna total del ozono estratosférico (Komhyr, 1980; Dobson, 1957). Igualmente, la R-UV que resulta ser sometida a fenómenos dispersivos por parte de los componentes del aire atmosférico (Murad E Tabocchini, 1976), también está involucrada en la determinación del ozono, como factor que disminuye la R-UV directa.

\section{MATERIALES Y MÉTODOS}

La tabla 1 muestra las series de datos reportados por Murad \& Tabocchini (1976), Komhyr (1980) y Bernhard et al. (2005b), para el coeficiente espectral de absorción del ozono en el rango espectral UV, entre 305,5 y 339,9 nm. Las series de Murad \& Tabocchini (1976) (sin asterisco), no reportan análisis de errores ni sus validaciones experimentales respectivas. Los datos mostrados con un asterisco (*) son los 
obtenidos por Komhyr (1980) y validados por observaciones realizadas en el Observatorio de Mauna Loa (Hawai, USA), para obtener datos del ozono total. La serie de valores reportada por Bernhard et al. (2005b), con doble asterisco (**), discrepan de los de Komhyr (1980), en $\pm 1,2 \%$ y fueron calculados asumiendo una temperatura de $-46.3^{\circ} \mathrm{C}$. A cada una de estas series de datos de los coeficientes espectrales de absorción del ozono, se les aplica un ajuste de funciones matemáticas y se plantea que un valor interpolado debería estar dentro de los respectivos ajustes de esas series de datos. Los datos reportados por son aceptados como los de la atmósfera estándar por el Climate Monitoring and Diagnostics Laboratory (CMDL). Es importante destacar que el CMDL mantiene un grupo de quince instrumentos Dobson y opera el Centro Mundial Dobson de Calibración del Ozono, bajo los auspicios del programa de Vigilancia Atmosférica Global de la Organización Meteorológica Mundial (OMM). Solamente, se dispone de la serie de datos del coeficiente espectral de dispersión molecular del aire, reportada por Murad E Tabocchini (1976), a la cual, se le hace el respectivo procedimiento.

Tabla 1. Coeficiente de absorción del ozono para longitudes de onda entre 0,3055 mm y 0,3399 mm.

\begin{tabular}{|c|c|c|}
\hline $\begin{array}{l}\text { Longitud de onda } \\
\qquad \lambda(\mu \mathrm{m})\end{array}$ & $\begin{array}{c}\text { Coeficiente de absorción } \\
\text { del ozono } \\
\mathrm{k}_{\mathrm{o \lambda}}\left(\mathrm{cm}^{-1}\right)\end{array}$ & $\begin{array}{c}\text { Coeficiente de dispersión molecular } \\
\text { de aire puro } \\
\mathrm{k}_{\mathrm{r} \lambda}\left(\mathrm{cm}^{-1}\right)\end{array}$ \\
\hline 0,3055 & 1,$882 ; 1,915 * ; 1,914 * *$ & 0,491 \\
\hline $\begin{array}{l}0,3088 \\
0,3089\end{array}$ & $\begin{array}{c}1,287 ; \\
1,239 * ; 1,242 * * \\
\end{array}$ & 0,470 \\
\hline $\begin{array}{l}0,3114 \\
0,3115\end{array}$ & $\begin{array}{c}0,912 ; \\
0,873^{*} ; 0,871\end{array}$ & 0,453 \\
\hline $\begin{array}{l}0,3176 \\
0,3175\end{array}$ & $\begin{array}{c}0,391 ; \\
0,384^{*} ; 0,387^{* *}\end{array}$ & 0,416 \\
\hline $\begin{array}{l}0,3250 \\
0,3254\end{array}$ & $\begin{array}{c}0,109 * ; 0,110 * * \\
0,120\end{array}$ & 0,375 \\
\hline 0,3291 & 0,$064 ; 0,062 * ; 0,063 * *$ & 0,357 \\
\hline 0,3324 & 0,$047 ; 0,040 * ; 0,039 * *$ & 0,343 \\
\hline $\begin{array}{l}0,3398 \\
0,3399\end{array}$ & $\begin{array}{c}0,017 ; \\
0,017^{*} ; 0,010^{* *}\end{array}$ & 0,312 \\
\hline
\end{tabular}

Con el propósito de tener elementos de juicio para verificar la confiabilidad de valores obtenidos por interpolación de las series de datos experimentales y la pertinencia de su uso para la determinación de la capa de ozono, se debe cuenta algunos criterios mínimos entre los cuales se citan:

Validación de aspectos instrumentales: Los que tienen que ver con la calibración de la respuesta espectral del instrumento y con la función de la apertura o colimación del haz de radiación. Para el caso de las series a tratar en el presente artículo, los autores de las mismas realizaron las correspondientes validaciones (Bernhard et al. 2005b), con la metodología estándar aplicable a todos los casos de calibración de radiómetros UV (Bernhard et al. 2005a). La serie de datos del coeficiente de dispersión del aire no serán validados por falta de información experimental adicional; sin embargo, un estudio futuro puede ser validado utilizando los resultados de este trabajo.

Condiciones experimentales: Referidas al entorno, como por ejemplo, la temperatura y la presión ambientales en las que se realizan las mediciones, dentro del cual, se caracterizan, tanto el ozono como el aire. Con la metodología aplicada a los espectrofotómetros Dobson, se observa que la obtención del ozono total está afectada por varios factores de error, relacionados con condiciones ambientales: 
- La omisión en las correcciones de la temperatura estratosférica puede conducir a errores de hasta $4 \%$, según Bernhard et al. (2005b). Se debe tener en cuenta, además, que las variaciones del perfil vertical del ozono afectan los valores reales del coeficiente espectral de absorción por parte del ozono. En las latitudes altas, las temperaturas estratosféricas están íntimamente ligadas a las variaciones estacionales (Goody, 1964).

- Las aproximaciones en la estimación de la masa de aire generan errores sistemáticos en el cálculo del ozono total (Redondas et al. 1996).

- Los errores se incrementan para condiciones de salida y puesta del Sol, ya que según Komhyr (1980), tiende a presentarse un aumento de la radiación espectral difusa, especialmente, en los rangos de bajas longitudes de onda, lo cual, se refleja en una aparente disminución del ozono total. En general, lo anterior también se aplica a grandes ángulos cenitales, es decir, a las regiones en las que el ángulo entre la vertical y la posición del Sol es grande, lo que, básicamente, se presenta en latitudes altas.

- Para disminuir efectos no deseables, como los de bruma, contaminación y nubosidad, se prefiere realizar las mediciones del ozono total en días despejados y libres de contaminación (Redondas et al. 1996).

Factores de error: Se evidencia ahora que los anteriores factores tienen menor peso para latitudes ecuatoriales debido a:

- Las temperaturas estratosféricas están menos afectadas por variaciones estacionales (Goody, 1964) y las correcciones requeridas serían mucho menores, por lo cual, valores de absorción del ozono y/o de dispersión del aire, ya sean experimentales o ya obtenidos por interpolación, contribuirán con errores sistemáticos mucho menores a $4 \%$.

- De manera natural, los ángulos cenitales son menores que en las grandes latitudes, lo cual, se hace aún más claro, si se tiene en cuenta que los periodos de medición del ozono total se ubican alrededor del mediodía.

- Aún con las anteriores condiciones favorables es muy importante tener en cuenta que las mediciones del ozono total se deben realizar, preferiblemente, en días despejados (Redondas et al. 1996).

Criterios estadísticos: Normalmente aplicados al comportamiento de las series de datos en cuestión, comparados con los estándar referenciales, a través de indicadores que definen rangos de error, desviaciones y/o correlaciones e índices de determinación; en su publicación, Bernhard et al. (2005b) contrastan sus cálculos con los resultados experimentales de Komhyr (1980).

Las tres series de datos antes mencionadas son comparadas respecto al coeficiente de absorción del ozono desde el punto de vista de un ajuste de línea de tendencia en función de la longitud de onda dentro del rango UV-B; luego, desde el mismo punto de vista, se realizará un análisis semejante para la única serie de datos del coeficiente de dispersión del aire puro.

Coeficiente de absorción del ozono en función de la longitud de onda: La información compilada en la tabla 1 muestra las tres series de datos relativas al coeficiente de absorción del ozono en función de la longitud de onda, de las cuales, se muestran sus curvas en la gráfica 1, donde se escogió, como ajuste de línea de tendencia, una función potencial. Cabe anotar que este ajuste fue escogido entre varios ajustes, en el que el coeficiente de correlación de los datos $\mathrm{R}^{2}$ tomó valores entre 0,995 y 0,998. Se observa que las tres series de datos muestran un comportamiento y una tendencia similares.

Las discrepancias de Bernhard et al. (2005b) y la serie de Komhyr (1980) están dentro de un error relativo de $\pm 1,2 \%$ y señalan que los datos obtenidos por interpolación son válidos para ser utilizados en cálculos del ozono total.

Coeficiente de dispersión molecular del aire puro: Se realiza el ajuste matemático solo para la serie de datos reportados por Murad E Tabocchini (1976) (Tabla 1).

En la gráfica 2, se ilustra el coeficiente de dispersión molecular del aire puro en función de la longitud de onda, para el rango UV-B. En este caso, se obtiene un excelente ajuste a una función polinómica de grado 2 con $\mathrm{R}^{2}$ de 0,997 . Puesto que no hay datos de referencia, se asumirá que una interpolación con esta serie podría arrojar resultados validables.

Corresponde a este trabajo acudir a alguna forma de validación de los resultados obtenibles del procedimiento propuesto, ya que las series de datos, base del estudio en este artículo, fueron validadas por los respectivos grupos de investigación en los artículos referenciados.

Una ruta natural de validación es aplicar los resultados obtenidos de los coeficientes de absorción del ozono y de dispersión del aire puro para mediciones de R-UV de longitudes de onda requeridas, en condiciones reales.

La Fundación Universitaria Los Libertadores, con sede en Bogotá, cuenta con un radiómetro ultravioleta GUV2511 de Biospherical Instruments Inc., instalado en el Laboratorio Radiométrico de la misma. Este equipo registra la R-UV espectral global en las longitudes de 305, 313, 320, 340, 380 y 395nm; dentro de este conjunto de longitudes de onda, se desconocen los coeficientes de absorción por ozono y de dispersión por aire de las líneas 313, 320 y 340nm, lo que motiva el interés de aplicar la metodología propuesta, para estos casos específicos. Nótese que las líneas de 380 y 395 $\mathrm{nm}$, se salen del rango de interés en este estudio. 


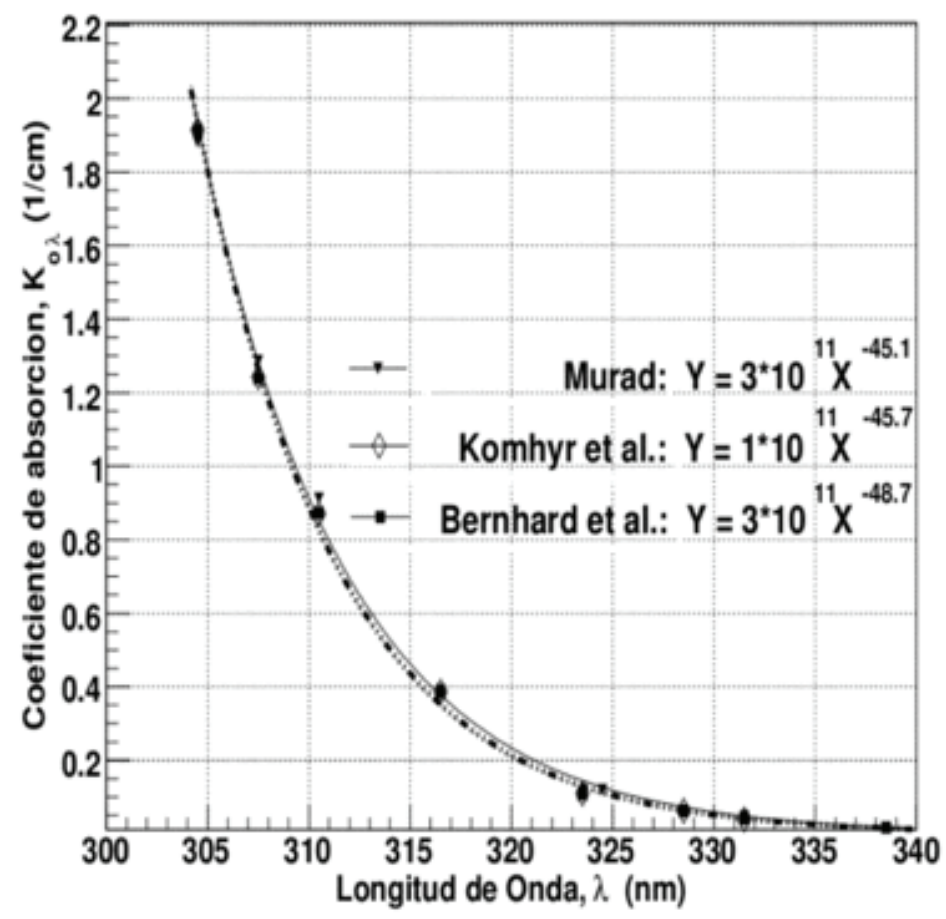

Gráfica 1. Coeficiente de absorción del ozono en función de la longitud de onda en el rango espectral, entre 305 y 340 nm, según las medidas reportadas por Murad E Tabocchini (1976) (triangulo invertido), Komhyr (1980) (rombo) y Bernhard et al. (2005b) (cuadrado).

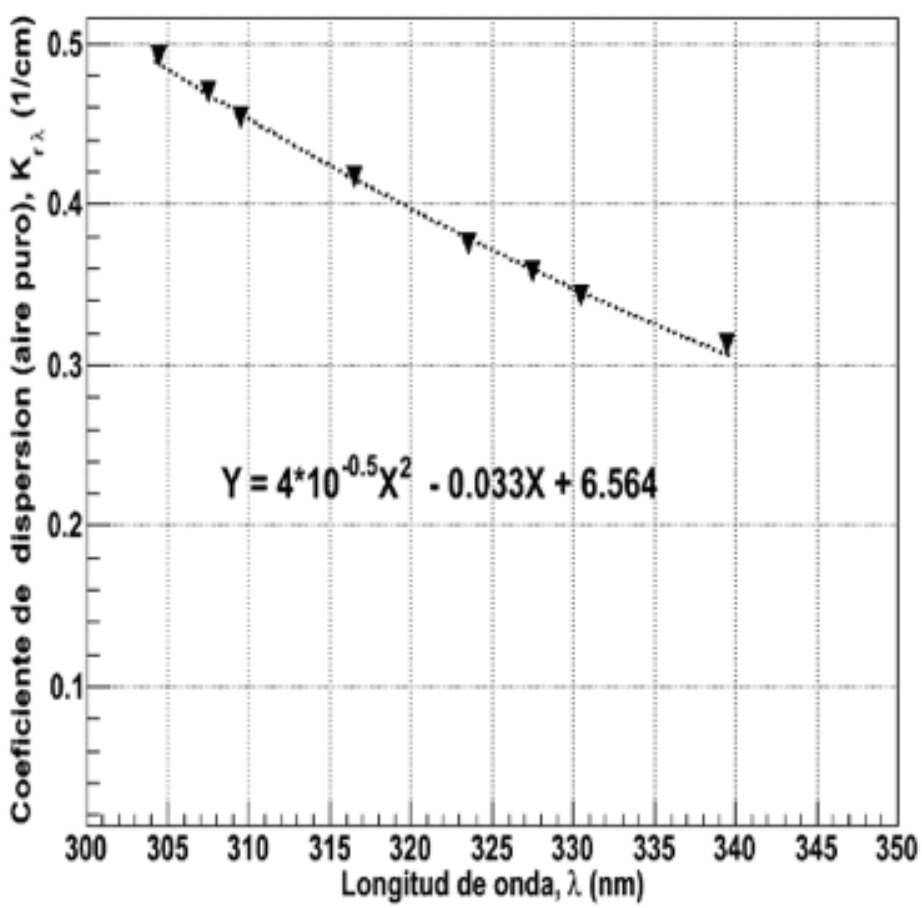

Gráfica 2. Coeficiente de dispersión molecular del aire puro en función de la longitud de onda en el rango espectral, entre 305 y $340 \mathrm{~nm}$, según las medidas reportadas por Murad E Tabocchini (1976). 
Como procedimiento de validación se recurrirá a una metodología altamente indirecta pero de alguna manera indicadora, ya que se trata de estimar la componente directa de la radiación espectral obtenida de la medición de la radiación global del equipo GUV2511, partiendo del dato del coeficiente de dispersión del aire puro (obtenido por interpolación). Cabe aclarar que el coeficiente se refiere al aire puro, y necesariamente se debe disponer de mediciones de R-UV global para días despejados y libres de contaminación; estas condiciones son bastante difíciles de alcanzar en la ciudad de Bogotá, razón por la cual, se trabaja con los resultados logrados en días semi-despejados, ya que además se enfrentaba el fenómeno de la Niña.
El valor de la radiación solar directa $\mathrm{I}_{\mathrm{D}}(\mathrm{l})$, se obtiene restando la componente difusa de la radiación global. La radiación difusa, $I_{d}(I)$, se consigue de multiplicar el valor de la R-UV global espectral $\mathrm{I}_{\mathrm{G}}(\mathrm{l})$, de las longitudes de onda en cuestión, por el valor respectivo del coeficiente de dispersión $\mathrm{k}_{\mathrm{rl}}$. Esto es:

$\mathrm{I}_{\mathrm{D}}(\lambda)=\mathrm{k}_{\mathrm{r} \lambda}\left[\lambda_{\mathrm{G}}(\lambda)\right]$

$\mathrm{I}_{\mathrm{D}}(\lambda)=\mathrm{I}_{\mathrm{G}}(\lambda)-\mathrm{k}_{\mathrm{r} \lambda}\left[\mathrm{I}_{\mathrm{G}}(\lambda)\right]=\mathrm{I}_{\mathrm{G}}(\lambda)\left[1-\mathrm{k}_{\mathrm{r} \lambda}\right]$

En la tabla 2, se consignan los valores de los coeficientes conseguidos por interpolación para las radiaciones ultravioleta 313, 320, 340nm y los resultados de la estimación

Tabla 2. Valores de los coeficientes obtenidos por interpolación para las radiaciones ultravioleta 313, 320, 340nm y los resultados de la estimación de la radiación directa, para el día 15 de mayo de 2010m entre la 11h30min y las 23h40min.

\begin{tabular}{|c|c|c|c|c|c|}
\hline $\begin{array}{l}\text { Longitud de } \\
\text { onda } \\
\lambda(\mathrm{nm})\end{array}$ & $\begin{array}{l}\text { Coeficiente de } \\
\text { absorción del } \\
\text { ozono } \\
\left(\mathrm{k}_{\mathrm{o} \curlywedge}\right)\end{array}$ & $\begin{array}{l}\text { Coeficiente } \\
\text { de dispersión } \\
\text { molecular de } \\
\text { aire puro } \\
\left(\mathrm{k}_{\mathrm{r} \lambda}\right)\end{array}$ & $\begin{array}{l}\text { Radiación } \\
\text { global } \\
\mathrm{i}_{g}(\lambda)(\mu \mathrm{w} / \\
\left.\mathrm{cm}^{2} \mathrm{~nm}\right)\end{array}$ & $\begin{array}{l}\text { Factor para } \\
\text { determinar } \\
\text { radiación } \\
\text { directa } \\
\left(1-\mathrm{k}_{\mathrm{r} \lambda}\right) \\
\end{array}$ & $\begin{array}{c}\text { Radiación } \\
\text { directa } \\
\text { estimada } \\
\mathrm{i}_{\mathrm{d}}(\lambda) \\
\left(\mu \mathrm{w} / \mathrm{cm}^{2} \mathrm{~nm}\right)\end{array}$ \\
\hline 313 & 0,742 & 0,439 & 31,96875 & 0,561 & 17,9345 \\
\hline 320 & 0,274 & 0,41 & 45,38178 & 0,59 & 26,7753 \\
\hline 340 & 0,015 & 0,3099 & 73,759843 & 0,6901 & 50,9017 \\
\hline
\end{tabular}

de la radiación directa, para el día 15 de mayo de 2010, entre la 11h30min y las 23h40min, día que se clasificó como semidespejado.

Debido a la restricción experimental para medir la componente directa de la radiación ultravioleta, mediante el GUV511, no es posible realizar una validación inmediata y directa de los datos estimados, por tal motivo, se recurre a un procedimiento indirecto, que consiste en aplicar estos datos en la estimación del ozono total. En consecuencia, el siguiente paso para incluir la validación de los datos estimados del coeficiente de absorción del ozono es llevar estos datos a la metodología de Dobson (Ecuación 5) y determinar el valor de la columna total de ozono; por ejemplo, para el día 15 de mayo de 2010 y comparar el dato obtenido con el valor satelital reportado en NASA (2011).

La metodología Dobson aquí aplicada está descrita en Murad E Tabocchini (1976), la cual, requiere de dos pares de longitudes de onda de R-UV B. Para este estudio, se definen así: par A, 305nm (onda corta) y 320nm (onda larga); par B, 313nm (onda corta) y 340nm (onda larga). La tabla 3 resume los correspondientes coeficientes y su preparación para aplicarlos en el método de Dobson y determinar el valor de ozono total, $\Omega$, calculado así:

$\Omega=\frac{\left[\log \left(\mathrm{I}_{\mathrm{o} \lambda \mathrm{d}} / \mathrm{I}_{\mathrm{o} \lambda \mathrm{l}}\right)-\log \left(\mathrm{I}_{\lambda \mathrm{c}} / \mathrm{I}_{\lambda \mathrm{l}}\right)\right]}{\left.\left[\left(\mathrm{k}_{\mathrm{oc}}-\mathrm{k}_{\mathrm{ol}}\right) \mathrm{m}_{\mathrm{o}}\right]-\left[\left(\mathrm{k}_{\mathrm{rc}}-\mathrm{k}_{\mathrm{rl}}\right) \mathrm{m}_{\mathrm{r}}\right] /\left[\mathrm{k}_{\mathrm{oc}}-\mathrm{k}_{\mathrm{ol}}\right) \mathrm{m}_{\mathrm{o}}\right]}$

donde $\mathrm{I}_{\lambda}$ es la irradiancia solar directa que incide en la superficie después de atravesar la atmósfera; $\mathrm{I}_{\mathrm{o}}$, la irradiancia solar extraterrestre con longitud de onda $\lambda$; los subíndices $c$ y l, se refieren a onda corta y larga, respectivamente para cada par Dobson; $\mathrm{m}_{\mathrm{o}}$, la masa óptica relativa de ozono y $\mathrm{m}_{\mathrm{r}}$ es el camino óptico o masa óptica del aire, atravesada por la radiación en su paso por la atmósfera. De acuerdo a Robinson (lqbal, 1983), $\mathrm{m}_{\mathrm{o}}$ se puede calcular de:

$\mathrm{m}_{\mathrm{o}}=\left(1+\mathrm{z}_{3} / \mathrm{r}_{\mathrm{e}}\right) /\left[\cos ^{2} \theta_{\mathrm{z}}+2\left(\mathrm{z}_{3} / \mathrm{r}_{\mathrm{e}}\right)\right]^{1 / 2}$ 
con $z_{3}$, la altura sobre el nivel del mar del sitio de medición; $\mathrm{r}_{\mathrm{e}}$, el radio medio de la Tierra y $\theta_{\mathrm{z}}$, el ángulo cenital correspondiente al sitio y la hora de la medición; $m_{r}$, según Kasten \& Young (1989), se puede calcular así:

$\mathrm{m}_{\mathrm{r}}=\left[\operatorname{Cos} \theta_{\mathrm{z}}+0.50572\left(96.07995-\theta_{\mathrm{z}}\right)^{-1.6364}\right]^{-1}$
Puesto que la irradiancia solar extraterrestre varía diariamente según la posición de la Tierra con respecto al Sol, se utiliza el valor de la constante solar espectral (Tabla 4) y se calcula el valor diario mediante procedimientos, como el reportado por Simbaqueva E González (2009).

Tabla 3. Constantes características de interacción atmosférica para cada una de las longitudes de onda que intervienen en los pares del método de Dobson, adoptado para el radiómetro GUV 2511, del Laboratorio Radiométrico de la Fundación Universitaria Los Libertadores.

\begin{tabular}{|c|c|c|c|c|c|c|c|}
\hline \multirow{3}{*}{$\begin{array}{l}\text { PAR } \\
\text { A }\end{array}$} & \multirow{2}{*}{\multicolumn{2}{|c|}{$\begin{array}{c}\text { Longitudes de } \\
\text { onda } \\
\lambda(\mathrm{nm})\end{array}$}} & \multirow{3}{*}{$\begin{array}{c}\text { Coeficientes de } \\
\text { absorción } \\
\mathrm{k}_{\mathrm{oc}} \\
\mathrm{k}_{\mathrm{ol}}\end{array}$} & \multicolumn{2}{|c|}{$\begin{array}{l}\text { Coeficientes de } \\
\text { dispersión }\end{array}$} & \multirow{3}{*}{$\begin{array}{c}\begin{array}{c}\text { Diferencia } \\
\text { coeficientes } \\
\text { de absorción } \\
\mathrm{k}_{\mathrm{oc}}-\mathrm{k}_{\mathrm{ol}}\end{array} \\
1,64\end{array}$} & \multirow{3}{*}{$\begin{array}{c}\begin{array}{c}\text { Diferencia } \\
\text { coeficientes } \\
\text { de absorciór } \\
\mathrm{k}_{\mathrm{rc}}-\mathrm{k}_{\mathrm{rl}}\end{array} \\
0,081\end{array}$} \\
\hline & & & & & & & \\
\hline & 305 & 320 & & 0,491 & 0,41 & & \\
\hline B & 313 & 340 & $0,7420,015$ & 0,439 & 0,3099 & 0,727 & 0,1291 \\
\hline
\end{tabular}

Tabla 4. Valores de distancia Tierra-Sol, irradiancias espectrales extraterrestres y longitudes de onda para el cálculo de pares Dobson.

\begin{tabular}{|c|c|c|}
\hline $\begin{array}{c}\text { Longitudes de onda } \\
\lambda(\mathrm{nm})\end{array}$ & $\begin{array}{c}\text { Irradiancia Espectral Extraterrestre } \\
\mathrm{I}_{\mathrm{sc}}\left(\mathrm{Wm}^{-2} \mathrm{~nm}^{-1}\right)\end{array}$ & $\begin{array}{c}\text { Corrección por excentricidad para el } \\
\text { día 15-05-2010 }(\mathrm{n}=135) \\
\mathrm{I}_{\mathrm{sc}}\left(\mathrm{R}_{\mathrm{d}} / \mathrm{R}\right)^{2}=\mathrm{I}_{\mathrm{sc}} 0,978\end{array}$ \\
\hline 305 & $0,595^{*}$ & 0,582 \\
\hline 320 & 0,712 & 0,696 \\
\hline 313 & $0,686^{* *}$ & 0,671 \\
\hline 340 & $0,964^{* * *}$ & 0,943 \\
\hline
\end{tabular}

Con los valores de la tabla 4 , se calculan los pares Dobson, obteniendo para $\Omega_{\mathrm{A}}=0.241$ y $\Omega_{\mathrm{B}}=0.212$. Siguiendo el método ilustrado por Murad E Tabocchini (1976), se mezclan los resultados de los pares anteriores en la forma siguiente:

$$
\Omega=\frac{\left(\mathrm{N}_{\mathrm{A}}-\mathrm{N}_{\mathrm{B}}\right)}{\frac{\left.\left[\left(\mathrm{k}_{\mathrm{oc}}-\mathrm{k}_{\mathrm{ol}}\right)_{\mathrm{A}}-\left(\mathrm{k}_{\mathrm{oc}}-\mathrm{k}_{\mathrm{ol}}\right)_{\mathrm{B}}\right] \mathrm{m}_{\mathrm{o}}\right]-\left[\left(\mathrm{k}_{\mathrm{rc}}-\mathrm{k}_{\mathrm{rl}}\right)_{\mathrm{A}}-\left(\mathrm{k}_{\mathrm{rc}}-\mathrm{k}_{\mathrm{rl}}\right)_{\mathrm{B}} \mathrm{m}_{\mathrm{r}}\right]}{\left.\left[\left(\mathrm{k}_{\mathrm{oc}}-\mathrm{k}_{\mathrm{ol}}\right)_{\mathrm{A}}-\left(\mathrm{k}_{\mathrm{oc}}-\mathrm{k}_{\mathrm{ol}}\right)_{\mathrm{B}}\right] \mathrm{m}_{\mathrm{o}}\right]}}
$$

siendo $\mathrm{N}_{\mathrm{A}}=\left[\log \left(\mathrm{I}_{\mathrm{O} \lambda d} / \mathrm{I}_{\mathrm{o} \lambda \mathrm{I}}\right)-\log \left(\mathrm{I}_{\mathrm{D} \lambda d} / \mathrm{I}_{\mathrm{DNI}}\right)\right]$, para los respectivos valores del par $A$ y $\mathrm{N}_{\mathrm{B}}=\left[\log \left(\mathrm{I}_{\mathrm{o \lambda d}} / \mathrm{I}_{\mathrm{o \lambda l}}\right)-\log \left(\mathrm{I}_{\mathrm{D \lambda d}} / \mathrm{I}_{\mathrm{D \lambda}}\right)\right]$, para los valores del par $\mathrm{B}$, obteniendo así un valor total de ozono de 252 UD (Unidades Dobson), para el día 15 de mayo de 2010.

\section{RESULTADOS Y DISCUSIÓN}

El valor total de ozono de 252 UD encontrado por este estudio para el día 15 de mayo de 2010, contrastado con el reporte de NASA (2011) de 240 UD, arroja una discrepancia de tan solo un 5\%. Se aclara que el valor tomado de NASA no reporta incertidumbre experimental y se toma como referente internacional; además, el día 15 de mayo de 2010, en la zona de medición (Sede Bolívar de la Fundación Universitaria Los Libertadores), las condiciones eran de cielo medianamente despejado y que a la hora de la medición, 11 h35min, se presenta una situación de alta contaminación. Para esta localidad, Simbaqueva y González (2009) reportan que el coeficiente de turbiedad de Ångström fluctúa a lo largo del día, comenzando en 0,200, a las nueve de la 
mañana y aumentando hasta 0,350, a las dos de la tarde; hacia el final de la tarde puede tomar valores aún mayores. Datos adicionales suministrados por la Secretaría Distrital del Ambiente SDA (2009), confirman tal comportamiento en esta localidad. Igualmente, no se disponen datos adicionales de otras variables meteorológicas para el día en mención y, por tanto, es imposible cuantificar el efecto de elementos no deseados en la incertidumbre del valor estimado. De todas formas, el valor estimado en las condiciones descritas debe presentar un sesgo hacia valores mayores que el reportado por NASA.

La vasta amplitud de las investigaciones que se realizan sobre el ozono requiere disponer de todas sus características espectrales; sin embargo, al no disponer de conjuntos completos de mediciones, es razonable recurrir a interpolaciones dentro de series discretas de datos, siempre y cuando éstas cumplan algunos criterios mínimos.

Inicialmente, poseer datos que provengan de mediciones rigurosas y que sean referentes ante la comunidad científica que las avala. Seguidamente, los datos se deben contrastar con las mediciones de otros grupos, en condiciones experimentales similares. Adicionalmente, es importante que los ajustes de línea de tendencia basados en modelos matemáticos y estadísticos muestren altos coeficientes de determinación. Por último, los datos deben soportar diferentes procesos de validación instrumental, física, estadística y matemática.

Respecto al rango espectral específico que refiere este trabajo, se evidencia que ciertas metodologías de medición de ozono, se pueden beneficiar al ampliar el conocimiento del comportamiento del ozono, en cuanto a la absorción de la R-UV B, en particular, cuando se integra la metodología Dobson a instrumentos diferentes a los espectrofotómetros Dobson. El beneficio más importante consiste en que aquellas metodologías que utilizan la radiación UV-B solar global tienen una forma de estimar la R-UV B solar directa a partir de este procedimiento; esto permite hacer investigaciones sobre el comportamiento y el efecto de las nubes y los aerosoles. Debido a la ubicación geográfica y a la gran incidencia de nubes cambiantes en el tiempo, se propone a la Sabana de Bogotá como un importante laboratorio para el estudio de las nubes y sus efectos sobre el ambiente.

Agradecimientos: Los autores de este trabajo agradecen a la Fundación Universitaria los Libertadores y, particularmente, a su Dirección de Investigación, por el soporte financiero al proyecto "Valoración de las Condiciones Atmosféricas que Determinan el Riesgo por Exposición a la Radiación Solar Ultravioleta en la Sabana de Bogotá", proyecto del cual, surge el presente artículo. Conflictos de intereses: El trabajo fue preparado por el primer autor y revisado y avalado por el segundo autor, quienes declaramos que no existe ningún conflicto de intereses que ponga en riesgo la validez de los resultados presentados.

\section{BIBLIOGRAFÍA}

1. BERNHARD, G.; BOOTH, C.R.; EHRAMJIAN, J.C. 2005a. Real-time ultraviolet and column ozone from multichannel ultraviolet radiometers deployed in the National Science Foundation's ultraviolet monitoring network. Optical Engineering. 44(4):041011-1-041011-11.

2. BERNHARD, G.; EVANS, R.D.; LABOW, G.J.; OLTMANS, S.J. 2005b. Bias in Dobson total ozone measurements at high latitudes due to approximations in calculations of ozone absorption coefficients and air mass. J. Geophys. Res. 110(D10):10.

3. BOSCÁ, J.V. 1995. Contribución al estudio de la radiación solar y de la determinación de la turbiedad atmosférica. Aplicaciones a Valencia y Sevilla. Tesis doctoral, Universidad Politécnica de Valencia (España). 393p.

4. DOBSON, G.M.B. 1957. Handbook for the Ozone Spectrophotometer Part I. Ann. Int. Geophys. 48-89.

5. GARCÍA, R.D. 2009. Análisis de la capacidad de los modelos de transferencia radiativa para la calibración de los radiómetros: aplicación al radiómetro NILU-UV. Nota Técnica Digital No. 4. Centro de Investigación Atmosférica de Izaña (España). 94p.

6. GOODY, R.M. 1964. Atmospheric Radiation. Clarendon Press (Inglaterra). 436p.

7. GREBENSHCHIKOV, S.YU.; QU, Z.-W.; ZHUZ, W.H.; SCHINKE, R. 2007. New theoretical investigations of the photodissociation of ozone in the Hartley, Huggins, Chappuis, and Wulf bands. Phys. Chem. Chem. Phys. 9:2044-2064.

8. IQBAL, M. 1983. An Introduction to Solar Radiation. Academic press (Canada). 377p.

9. KASTEN, F; YOUNG, T. 1989. Revised optical air mass tables and approximation formula. Applied Optics 28:4735-4738. 
10. KOMHYR, W.D. 1980. Operations Handbook - Ozone Observations with a Dobson Spectrophotometer. Prepared for the World Meteorological Organization Global Ozone Research and Monitoring Project. Revised September, 2008 by Robert Evans. NOAA/ ESRL Global Monitoring Division. WMO/TD-No. 1469 (Suiza). 93p.

11. LIOU, K.N. 2002. An Introduction to Atmospheric Radiation. 2nd ed. Academic Press, San Diego (USA). 583p.

12. MURAD, A.C.; TABOCCHINI, H.L. 1976. Variaciones del Espesor de la Ozonósfera sobre Buenos Aires y su Relación con las Condiciones Meteorológicas de la Troposfera y la Estratosfera. Fuerza Aérea Argentina, Comando de Regiones Aéreas, Servicio Meteorológico Nacional (Argentina). 48p.

13. NATIONAL AERONAUTICS AND SPACE ADMINISTRATION (NASA) 2011. Total Ozone Mapping Spectrometer. Disponible desde Internet en: http:// toms.gsfc.nasa.gov/ozone/ozone.html (con acceso el 25/06/2011).

14. REDONDAS, A.; CUEVAS, E.; SANCHO, J.M. 1996. Investigación y Programa de Medidas del Ozono Estratosférico y la Radiación Ultravioleta en el Observatorio de Vigilancia Atmosférica de Izana (INM). Madrid. Física de la Tierra. 9:29-66.
15. SECRETARÍA DISTRITAL DEL AMBIENTE SDA. 2009. Informe anual de calidad del aire de Bogotá año 2008. Red de Monitoreo de Calidad del Aire de Bogotá D.C. Disponible desde Internet en:http:// www.secretariadeambiente.gov.co/sda/libreria/pdf/ InformeAnual/ Informe2008.pdf (con acceso 25/06/2011).

16. SIMBAQUEVA, O.; GONZÁLEZ, J.C. 2009. Un método y programa de cálculo para estimar turbiedad atmosférica sobre la Ciudad de Bogotá, utilizando medidas de radiación solar directa con pirheliómetro Eppley en todo el ancho de banda del espectro solar para días despejados. Proceedings Congremet $\mathrm{X}$, Climet XIII, Buenos Aires, Argentina.

17. STAEHELIN, J.; KERR, J.; EVANS, R.; VANICEK, K. 2003. Comparison of total ozone measurements of Dobson and Brewer spectrophotometers and recommended transfer function. World Meteorological Organization Global Atmosphere Watch WMO TD. 1147:1-35.

18. WORLD CLIMATE RESEARCH PROGRAMME (WCRP) 1986. World Meteorological Organization. Publications Series No. 7. WMO/TD No. 149. 39p.

Recibido: Marzo 22 de 2011

Aceptado: Noviembre 4 de 2011 